\title{
A NEW PROOF OF THE BONNICE-KLEE THEOREM
}

\author{
JOHN R. REAY
}

The purpose of this note is to give a new (and much easier) proof for a theorem of Bonnice-Klee [1]. Their result (Theorem 1 below) has proved to be quite useful in establishing a number of results concerning the convex hulls of certain sets (see [4]). It is a generalization of the following two classic results due respectively to Carathéodory and Steinitz.

(a) If $X$ is a subset of an $n$-dimensional real linear space $E^{n}$, and $p \in \operatorname{con} X$ (the convex hull of $X$ ), then $p \in$ con $U$ for some subset $U$ of $X$ with card $U$ (cardinality of $U$ ) at most $n+1$.

(b) If $X \subset E^{n}$, and $p \in$ int con $X$ (the interior of con $X$ ), then $p \in$ int con $U$ for some subset $U$ of $X$ with card $U \leqq 2 n$.

The generalization is based on the notion of intermediate interiors. The $d$-interior of a set $X \subset E^{n}$ (denoted by int ${ }_{d} X$ ) is the set of all points $p$ such that $p$ is in the relative interior of some $d$-simplex contained in $X$; equivalently, $p \in$ int $_{d} X$ iff there exists a $d$-dimensional flat $F$ through $p$ such that $p$ is in the interior of $X \cap F$ relative to $F$. The result may be stated as

THEOREM 1. If $X \subset E^{n}, 0 \leqq d \leqq n$, and $p \in$ int $_{d} \operatorname{con} X$, then $p \in$ int $_{d}$ con $U$ for some subset $U$ of $X$ with card $U \leqq \max (n+1,2 d)$.

Theorem 2 below was used in [4] as a tool to establish certain generalizations of the above result of Steinitz, and has also been used to obtain uniquely-defined continuous representations of points in $E^{n}$ in terms of an arbitrary positive basis. We will show that Theorem 1 of Bonnice-Klee is an easy consequence of Theorem 2. Set $B C E^{n}$ positively spans $E^{n}$ if each point of $E^{n}$ is a positive combination (i.e., a linear combination with non-negative coefficients) of the points of $B$. The set $B$ is a positive basis of $E^{n}$ if it is also positively independent, i.e., no element of $B$ is a positive combination of the remaining elements of $B$. (See [2], [3].) The positive hull of set $X$, denoted by pos $X$, is the set of all positive combinations of $X$.

THEOREM 2. Let $B$ be any positive basis for $E^{n}$. Then $B$ admits a partition into pair-wise disjoint subsets $B=B_{1} \cup \ldots \cup B_{k}(1 \leqq k \leqq n)$, such that $\operatorname{card} B_{i} \geqq \operatorname{card} B_{i+1} \geqq 2, \quad i=1, \cdots, k-1, \quad$ and $\operatorname{pos}\left(B_{1} \cup \ldots \cup B_{j}\right)$ is a linear subspace of $E^{n}$ of dimension $\left(\sum_{i=1}^{j} \operatorname{card} B_{i}\right)-j$ for $j=1,2, \cdots, k$.

Presented to the Society, June 20, 1964; received by the editors May 16, 1964. 
We will sketch the proof of Theorem 2, since certain parts of the machinery are needed in proving Theorem 1 . It is well known that if $B$ is a positive basis for an $n$-dimensional space $E$, then $n+1 \leqq$ card $B$ $\leqq 2 n$. Set $B$ is said to be minimal provided that card $B=n+1$. A subset $B^{\prime}$ of an arbitrary positive basis $B$ is in general not a positive basis for a linear subspace. Following the notation of McKinney [3], we say that a linear subspace $F$ of $E$ is a spanned subspace with respect to $B$ iff $F \cap B$ is a positive basis for $F$. If, moreover, $F \cap B$ is a minimal positive basis for $F$, then we say that $F$ is a minimal subspace (with respect to $B$ ). Davis [2] has shown that for each element $b$ of a positive basis $B$, there exists a set $B^{\prime}$ (not necessarily unique) such that $b \in B^{\prime} \subset B$ and pos $B^{\prime}$ is a minimal subspace of $E$ with respect to $B$. Thus $E$ always has at least one minimal subspace with respect to $B$.

Proof of Theorem 2. Let $B$ be a given positive basis for the space $E$, and let $B_{1}$ be a subset of $B$ of maximal cardinality such that $B_{1}$ is a minimal positive basis for the minimal subspace pos $B_{1}$. If $B=B_{1}$, the theorem is clear. Otherwise, let $E_{1}$ be a linear subspace of $E$ such that $E=E_{1} \oplus$ pos $B_{1}$ ( $\oplus$ means direct linear sum), and let $\pi_{1}$ be the natural projection of $E$ on to $E_{1}$. It follows that $\pi_{1}\left(B-B_{1}\right)$ is a positive basis for $E_{1}$. Also, if $B_{2}$ is a subset of $B-B_{1}$ of maximal cardinality such that $\pi_{1} B_{2}$ is a minimal positive basis for the minimal subspace pos $\pi_{1} B_{2}$ of $E_{1}$, then it follows that card $B_{1} \geqq \operatorname{card} B_{2}$ and $\operatorname{pos}\left(B_{1} \cup \pi_{1} B_{2}\right)$ $=\operatorname{pos}\left(B_{1} \cup B_{2}\right)$. (See [4] for details of these arguments.) It is clear that card $B_{1} \geqq \operatorname{card} B_{2} \geqq 2$ and $\operatorname{pos}\left(B_{1} \cup B_{2}\right)$ is a linear space of dimension card $B_{1}+$ card $B_{2}-2$. If $B_{1} \cup B_{2}=B$ the theorem is established. Otherwise the same argument may be applied to the space $E_{1}$ and the process repeated until $B=B_{1} \cup \cdots \cup B_{k}$, thus establishing Theorem 2.

Proof of Theorem 1. Suppose $X \subset E^{n}, 0 \leqq d \leqq n$, and $p \in$ int $_{d}$ con $X$. With no loss of generality we may suppose that $p=0$. Let $F$ denote the largest linear space contained in pos $X$, and let $m$ be the dimension of $F$. Then $m \geqq d$ because $0 \in$ int $_{d}$ con $X$. It follows from the maximality of $m$ that $(\operatorname{con} X) \cap F=\operatorname{con}(X \cap F)$. Thus we may restrict our attention to the set $X \cap F$ in the linear space $F$, or equivalently, we may assume that pos $X=E^{n}$. That is, $X$ positively spans $E^{n}$. Choose a subset $B$ of $X$ that is a positive basis for $E^{n}$, and let $B=B_{1} \cup \ldots \cup B_{k}$ be a partition of $B$ as in Theorem 2. Then $B_{1}$ is a minimal positive basis in $B$ of maximal cardinality. Thus card $B_{1}$ $\leqq n+1$. If $d<$ card $B_{1}$ then $0 \in$ int $_{d}$ con $B_{1}$ and we set $U=B_{1}$, establishing Theorem 1. For the case of $d \geqq$ card $B_{1}$, let $U=B_{1} \cup B_{2} \cup \ldots \cup B_{j}$ where $j$ is the least integer such that the dimension of the linear space $\operatorname{pos}\left(B_{1} \cup B_{2} \cup \cdots \cup B_{j}\right)$ is at least $d$. It is clear that $0 \in$ int $_{d}$ con $U$, 
and it only remains to show that card $U \leqq \max (n+1,2 d)$. Note that $d>\operatorname{dim} \operatorname{pos}\left(B_{1} \cup \ldots \cup B_{j-1}\right)=\left(\sum_{i=1}^{j-1} \operatorname{card} B_{i}\right)-(j-1)$. Thus card $U$ $=\sum_{i=1}^{j}$ card $B_{i}<d+(j-1)+\operatorname{card} B_{j}$ and it suffices to show that $(j-1)+\operatorname{card} B_{j} \leqq d+1$. But

$d \geqq\left(\sum_{i=1}^{j-1} \operatorname{card} B_{i}\right)-(j-1)+1 \geqq \operatorname{card} B_{1}+2(j-2)-(j-1)+1$

$=(j-1)+\operatorname{card} B_{1}-1$.

So $d+1 \geqq(j-1)+$ card $B_{j}$, which shows that card $U \leqq 2 d$ and thus establishes Theorem 1.

\section{REFERENCES}

1. W. Bonnice and V. L. Klee, The generation of convex hulls, Math. Ann. 152 (1963), 1-29.

2. C. Davis, Theory of positive linear dependence, Amer. J. Math. 76 (1954), 733-746.

3. R. L. McKinney, Positive bases for linear spaces, Trans. Amer. Math. Soc. 103 (1962), 131-148.

4. J. R. Reay, Generalizations of a theorem of Caratheodory, Mem. Amer. Math. Soc. No. 54 (1965).

Western Washington State College 